\title{
On Cloud-Centric Network Architecture for Multi-Dimensional Mobility
}

\author{
Kyu-Han Kim Sung-Ju Lee Paul Congdon \\ Networking \& Communications Laboratory \\ Hewlett-Packard Laboratories, Palo Alto, CA 94304, U.S.A. \\ \{kyu-han.kim, sjlee, paul.congdon\}@hp.com
}

\begin{abstract}
Despite pervasive deployment of wireless networks, maintaining seamless mobile connectivity within a set of local devices and to the remote cloud is still challenging. The crux of this challenge stems from the simultaneous interplay of multiple dimensions of a user's mobility - users frequently move between multiple access networks, mobile devices and unique personas. We identify new trends and challenges in providing rich mobile connectivity to mobile users. We then propose a novel Cloud-centric Architecture for Rich Mobile Experience Networking, called Carmen. Carmen is a distributed system that manages the mobile connectivity of a set of devices belonging to a particular individual, which we call the mobile personal grid (MPG). Carmen enables the MPG to efficiently collect context from a mobile user and coordinate key system resources across the MPG and cloud. We present new design principles and functional components of Carmen. In addition, we show our system prototype of Carmen's resource monitoring infrastructure to demonstrate its feasibility and benefits in improving the mobile user's networking experience.
\end{abstract}

\section{Categories and Subject Descriptors}

C.2.1 [Computer-Communication Networks]: Network Architecture and Design-Wireless Communication

\section{General Terms}

Design, Performance, Algorithms, Measurement, Experimentation

\section{Keywords}

Mobile networks, cloud-assisted connectivity service, wireless access networks, mobile personal grid (MPG)

Permission to make digital or hard copies of all or part of this work for personal or classroom use is granted without fee provided that copies are not made or distributed for profit or commercial advantage and that copies bear this notice and the full citation on the first page. To copy otherwise, to republish, to post on servers or to redistribute to lists, requires prior specific permission and/or a fee.

MCC'12, August 17, 2012, Helsinki, Finland.

Copyright 2012 ACM 978-1-4503-1519-7/12/08 ...\$10.00.

\section{INTRODUCTION}

As numerous cloud-based applications and services have been introduced for mobile users, there are increasing pressures and demands on persistent connectivity between mobile devices and the cloud. For example, cloud-based mobile gaming (e.g., OnLive [1]) and file sharing (e.g., dropbox [2]) are becoming popular, but place strong requirement on network connectivity.

However, mobility-induced dynamics across mobile devices, network infrastructure and cloud services inherently impede seamless interworking across heterogeneous access networks, and further limit the movement of content across multiple devices and the cloud. These limitations become even more pronounced as mobile users use multiple cloudconnected devices that increasingly interact with sensors and other embedded computing resources, through both public and private networks. We call such a computing environment the mobile personal grid (MPG).

In the MPG, maintaining and managing seamless connectivity is challenging. Mobile users frequently switch between multiple access networks (both public and private), interact with multiple devices (phone, tablet, laptop), and transition between multiple contextual environments (business, personal). Transitioning across all these dimensions simultaneously causes connectivity disruptions, increases device complexity, degrades network performance, and interrupts access to content. Existing technologies, such as personalarea networks (PANs) and the Digital Living Network Alliance (DLNA) [3], have addressed some of the challenges, but not the multiple dimensions of mobility simultaneously.

We propose Carmen, a Cloud-centric Architecture for Rich Mobile Experience Networking, to address these challenges. Carmen takes a holistic yet converged approach by rethinking the relationship between (i) the mobile device networking protocol stack, (ii) the wireless network infrastructure, and (iii) the cloud. These three components are tightly coupled and dynamically interact with each other. They jointly monitor and analyze the mobile environment, control and support the virtualized network resources for interworking with public networks, and adaptively share resources located in the MPG. Moreover, the cloud maintains the meta-states of the MPG's connectivity and provides the services of a personal connectivity assistant, which we call Avatar. The user's Avatar performs computationally intensive tasks on behalf of mobile devices and/or network infrastructure to improve the user's mobile network experience. 
Carmen requires comprehensive and accurate resource information from a user's MPG to make better decisions on connectivity, and we show the prototype of Carmen's resource monitoring infrastructure, as a work-in-progress. It monitors connectivity states among devices within the MPG and reports their results to the cloud. We are implementing it using Android OS, extensible messaging and presence protocol (XMPP), and a Linux-based server.

The rest of this paper is organized as follows. Section 2 motivates the need for seamless bonding between the mobile and the cloud, and identifies the challenges to meet this need. Section 3 presents new design principles to solve the challenges. Section 4 describes the proposed Carmen architecture. Section 5 shows our work-in-progress - the Carmen resource monitoring infrastructure. Section 6 discusses related work and Section 7 concludes with future work.

\section{MOTIVATION}

This section motivates the needs of seamless connectivity to mobile cloud services, and presents key challenges that mobile users face to maintain such connectivity.

\subsection{Persistent Bonding with Cloud}

Despite the rapid advance in the computing capability of mobile devices, resources are always limited. With rich resources available in the cloud, mobile users are increasingly depending upon persistent connectivity to cloud-based services, data, and contacts. This need is accelerating as mobile carriers and enterprise network operators expand the deployment of broadband and wireless networks. For example, most of the mobile operators plan to make major investments in the deployment of high-speed cellular data networks (4G or LTE), and LTE subscribers are expected to grow rapidly (e.g., $11 \mathrm{M}$ in 2011 to $422 \mathrm{M}$ in 2015) [4].

A recent survey [5] shows media-rich mobile applications, such as 3D mobile gaming, video and voice, are unprecedentedly dominant in mobile data traffic. Such applications require reliable connectivity to the cloud as well as relatively low network latency (e.g., <100 ms for mobile gaming). Moreover, mobile interactive applications (e.g., mobile augmented reality, mobile visual search) impose higher bandwidth and lower latency requirements from wireless access networks to cloud service.

The close bonding between the mobile devices and the cloud becomes more instrumental as users depend upon their personal computing devices for work, private, and social life. For instance, many workers use their personal smartphone or tablet to connect to enterprise infrastructure. Such a phenomenon, called Bring-Your-Own-Device (BYOD) or the consumerization of IT, is inevitable in the enterprise, campus and other mobile environments. Mobile users expect to have disruption-free connectivity of their personal devices across heterogeneous access networks and services.

\subsection{Trends and Challenges}

While we witness the wide deployment of wireless access networks, maintaining a persistent bond with the cloud remains challenging. Below, we discuss several trends that lead to these challenges.

- Multiple access networks: Mobile devices are equipped with multiple network interfaces, including Wi-Fi, Bluetooth, 3G/4G, NFC (Near Field Communication). De-

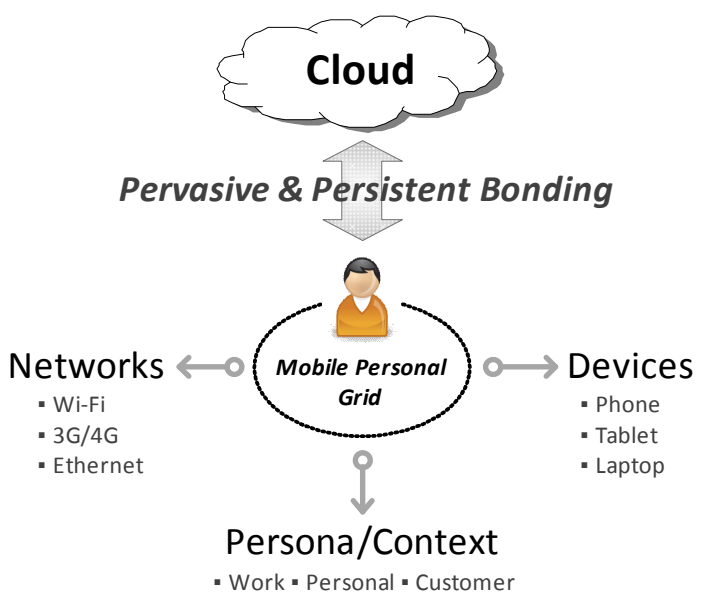

Figure 1: Mobile users faces multi-dimensional mobility-multiple access networks, devices, and personas - in mobile network connectivity to achieve close bonding with the cloud.

pending on a user's location, different access networks with different objectives (e.g., power saving, latency, throughput) may be used. Frequent switching at the end points causes service disruption, network performance degradation, and (manual) re-authentication.

- Multiple devices: Mobile users increasingly carry multiple mobile devices such as a smartphone, a tablet, and a laptop. In addition, the users can often interact with external computing devices [6] (big screen, entertainment systems, embedded/wearable sensors, etc.). Simultaneously working across different computing devices inherently disrupts a user's attention and service quality, especially when the user wants to migrate content and sessions across those devices. Furthermore, if mobile devices are equipped with different mobile platforms (software/hardware), it is difficult to maintain the same quality of experience.

- Multiple personas: Most mobile users carry one or more devices into different environments and context during their daily life. Imagine Bob, who has children (parental persona), works on sales (employee persona), and is active in social and personal activities (public persona). For each of his personas, Bob might need different network connectivity. For the work, he needs a secure and reliable connection during his customer meeting. On the other hand, for the public persona, his social application might get by with less bandwidth but requires privacy-preserving connectivity.

Figure 1 depicts these challenges. At any one moment, a mobile user may be moving across all of these dimensions of mobility simultaneously, creating a challenging environment for a persistent bonding with resources in the cloud.

\section{DESIGN PRINCIPLES}

To address the above challenges and provide a seamless multi-dimensional mobile network experience, we argue for the following design principles that impact the architecture of mobile device, wireless infrastructure and cloud. 


\subsection{Cloud-Centric Control}

Assuming that a mobile user has at least one (intermittent) connection to the Internet and/or her cloud at any given time, the cloud-centric connectivity control and assistant (Avatar) can provide several benefits over individual mobile-centric control in managing dynamic and heterogeneous environment within the MPG. These benefits include:

- Low complexity: For each connection that a user initiates, the MPG must handle multi-dimensional complexity; in addition to the changing network conditions accustomed to a wireless environment, the user's behavior and demands need to be monitored, aggregated, analyzed and authorized for her context. Juggling all these tasks would be an expensive operation in resource-constrained mobile devices, but the MPG can leverage the computing and storage resource of the cloud to keep this complexity in check.

- Accessibility: It is not uncommon for devices (even when placed side-by-side) to be unable to communicate directly with each other because they might be connected to different access networks (e.g., Wi-Fi vs. Cellular). However, the common global cloud is accessible from any interface that has Internet access. This commonality and global presence can be used to enable communicating and coordination of the MPG. Furthermore, persistent states of the MPG that are maintained in the cloud by Avatar allow for scheduled operations even when devices of the MPG are disconnected or are physically distant.

- Provisioning: While existing mobile-centric approach (e.g., [7]) or distributed approach (e.g., home agent of Mobile IP [8]) might have an advantage accessing mobile and network conditions, they are physically distant from the control points of services in the cloud. These control points are where quality provisioning takes place. Proxy-based approach (e.g., [9, 10]) can accelerate control, but it might not fully leverage the computing resource available in the cloud.

- Visibility: The Avatar is aggregating context and environmental data from all devices in the MPG and can make them available to all devices of the MPG as needed. Additionally, from the cloud vantage point, the Avatar can access historical and planned events (e.g., calendar) to provide an enhanced perspective on needed operations and actions for the MPG.

\subsection{Converged Access Networks}

There are growing demands to support seamless interworking between heterogeneous wireless access networks, particularly as mobile users increasingly bring their personal device(s) to workplace and conversely do more work on those devices in public areas. Enterprise network administrators are under pressure to allow external wireless access (e.g., cellular networks) while mobile operators are looking for ways to offload cellular traffic to Wi-Fi to provide better bandwidth and service. Converging network architectures creates opportunities with the following benefits:

- Context-aware interworking: Different radios in a mobile device can be associated with different administrative domains (e.g., Wi-Fi to the enterprise and $3 \mathrm{G}$ to the mobile operator). This separation of administrative domain has made it difficult to support offloading and seamless migration of connection-oriented flows. Recently, mobile operators, the Wi-Fi alliance, and network equipment manufacturers have initiated a converged architecture for the next-generation $\mathrm{Wi}$ Fi (or HotSpot 2.0 [11]). This converged architecture allows users to authenticate to each access network and share heterogeneous network resources. Moreover, each network can exchange and advertise its current network resources (bandwidth, latency, other services) to allow context-aware switching decisions.

- Interaction with cloud: Imagine that an access point (AP) interacts with a user's Avatar to understand the user's network activity, application usage, and history (e.g., traffic cache). The Avatar can pre-populate and provide resources based upon the information, so the network can better support the mobile user. For example, the Avatar can inform the network infrastructure of session migration for the mobile user, even before the user physically moves into the network's coverage. Such close interaction can create an environment in which a mobile user feels as if the cloud is locally attached with low latency. Finally, the network infrastructure can offload complex network tasks (e.g., configuration, traffic analytics) to the cloud.

\subsection{Resource Virtualization}

Moving between personas (i.e., contextual mobility) likely requires changes to the instantaneous amount of network resource in use. Similarly, moving between access networks (i.e., physical mobility) can induce changes to resource availability and operating conditions. Given the dynamic needs and supply of the network resource, a mobile user can benefit from resource virtualization as follows:

- Mobile device virtualization: As a mobile user physically moves, she can encounter various computing and network resource of heterogeneous type, capability and availability. In the MPG, each device may have different degrees of mobility and various levels of connection resources supporting different qualities of service. For example, in one location, only one device might have Internet connection (e.g., cellular) and in other locations, more than two devices might have connections (e.g., cellular, Wi-Fi). However, in either scenario, the user's application running under a particular persona in the MPG might require a persistent Internet connection. Virtualization of the mobile networking stack can abstract these dynamic mobile resources, allowing them to be shared to accommodate the different requirements as elements move around within the MPG.

- Access network virtualization: Network infrastructure, such as Wi-Fi access networks, can be virtualized. Many of Wi-Fi APs today support virtual AP functionality. For example, one AP can advertise multiple SSIDs (e.g., guest, employee). To address the needs of BYOD, network operators would like mobile users to align each application or each persona with a particular virtualized networks to avoid compromising security. This requires access networks to support fine-grained virtualization and isolation per persona or per application for each device in the MPG. 


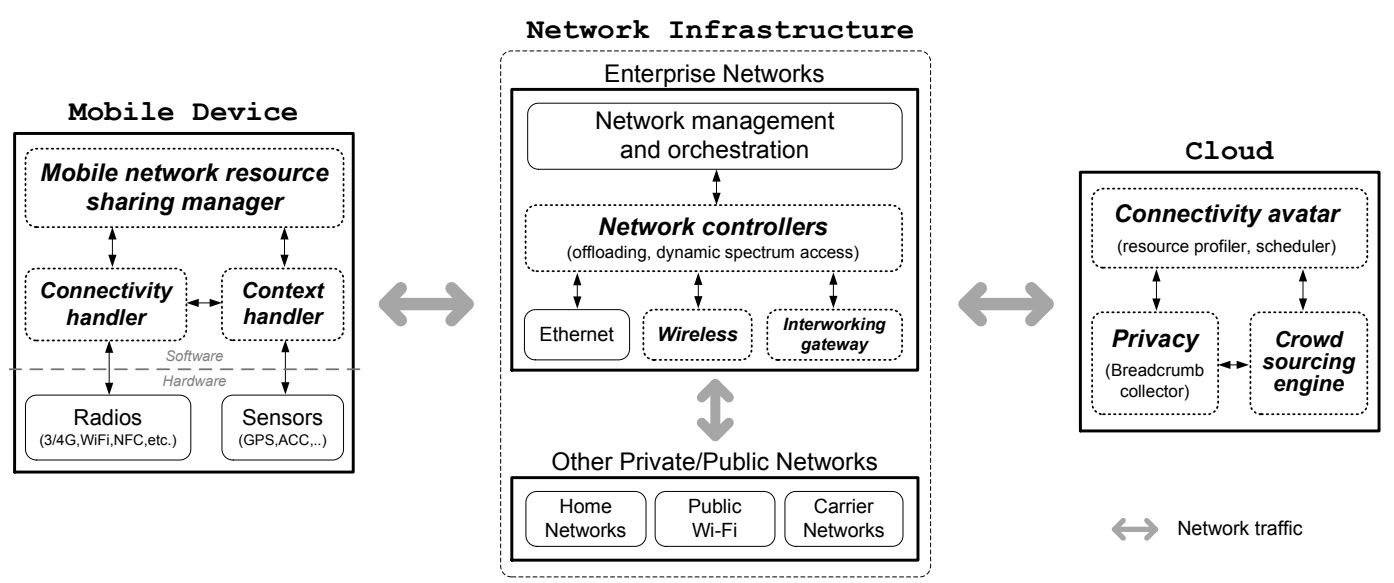

Figure 2: The Carmen architecture. Carmen takes a holistic approach across mobile devices, network infrastructure, and the cloud to provide rich mobile network experience to users.

\section{THE CARMEN ARCHITECTURE}

Figure 2 shows the architecture of Carmen. Carmen is a distributed system that allows for the formation and operation of the MPG, close interaction of the MPG with network infrastructure, and cloud-centric connectivity control maintained by Avatar in the cloud. In what follows, we detail the components and technical challenges.

\subsection{Mobile Devices}

On the mobile device, a light-weight monitoring and management system (connectivity handler) and user context system (context handler) provide the critical function of delivering personalized and context-aware services to the user. Next, a cloud-assisted mobile network resource sharing manager at the mobile device allows for discovery and management of network resources. Users create, modify, transfer, and consume the same content on multiple devices. Such activities require different levels of resources, but different devices are faced with different limitations (e.g., networks, computation, memory). The resource sharing manager enables fine-grained network and resource virtualization given heterogeneous hardware platforms (display, sensors).

Mobile devices and smartphones are equipped with a variety of sensors, including GPS, accelerometers, camera, and magnetic compasses, that provide indications of the device's current location, orientation and communications environment. However, it is challenging for application developers to take advantage of these capabilities. Our architecture includes a sensor-connectivity abstraction layer across both distributed and embedded sensors with open APIs that allow sensor owners to expose secure sensing capability to application developers.

\subsection{Access Network Infrastructure}

The network infrastructure is a critical component of the overall connection service quality. Network infrastructure that mobile users associate with will be heterogeneous and a novel network controller allows for seamless interworking across the heterogeneous networks (i.e., enterprise networks, cellular networks). Further, it includes a dynamic spectrum management framework that acts as a local spectrum broker and performs spectrum sensing/allocation and mobility support for wireless networks.
The key challenge for the network infrastructure is to transform one physical access network into multiple virtual, compliant and isolated networks such that each mobile device in the MPG obtains the necessary connectivity. Network virtualization techniques (e.g., OpenFlow [12]) can extend from the wireless access infrastructure directly into the mobile device to allow a logical slicing of resources for different groups of devices. An interworking gateway architecture provides easy integration of $\mathrm{Wi}-\mathrm{Fi}$ networks with carrier networks for Wi-Fi offloading, network resource sharing, and session management (e.g., admission control, security).

\subsection{Cloud}

By using monitoring results from the mobile devices and control knobs available in the network infrastructure, a cloudbased connectivity-assistant or Avatar facilitates various decisions on resource utilization, content sharing, and privacy, while enabling seamless network connectivity.

With the supporting network infrastructure, the Avatar addresses several key challenges. First, it establishes the means to easily form a trusted MPG from a set of uncoordinated devices; a device should only need to maintain a control channel with the Avatar to deliver credentials and connectivity parameters. Next, it coordinates pervasive content access across all devices in the MPG. It can use its global knowledge to determine the context and coordinate the connectivity as required. Finally, the Avatar can make use of profile and other contextual information about the user to better deliver content and provide improved connectivity. Moreover, as the Avatar is uniquely associated with the mobile user, the personal breadcrumb collector provides a secure and privacy-preserving mechanism.

Finally, our cloud architecture also includes a crowd sourcing engine that interacts with the context handler at the mobile device to provide users with the ability to handle contextual information.

\section{RESOURCE MONITORING OF MPG}

This section presents our work-in-progress to demonstrate the feasibility of Carmen. We explain Cloud-assisted remotemonitoring infrastructure for mobile networks, called Clarinet, and show our prototype in progress. 


\subsection{Design Goal}

Following the Carmen architecture, Clarinet is designed to monitor the network connectivity and resource availability within the MPG and share the monitoring information with mobile cloud-based applications or services.

\subsubsection{Managing dynamic MPG}

$\mathrm{Clarinet}$ is a remote monitoring infrastructure that creates and manages the MPG of each mobile user. Further, it maintains an Avatar instance for each MPG in the cloud to keep updated on resource information of the MPG. These functions incur a broad set of challenges. First, as devices in the MPG are dynamic and their radios are heterogeneous, formation and operation of the MPG needs to be deliberately configured. For instance, if one device is using a $\mathrm{Wi}-\mathrm{Fi}$ radio for Internet connection, the MPG might need to virtualize the radio into two logical radios - one for Internet and one for the MPG - or, use other radios such as Bluetooth to form a network among other MPG devices. Here, the MPG network is a one-hop topology from its leader node.

Another function of Clarinet is the creation of the Avatar instance in the cloud for the MPG. Once the MPG is locally created, its Avatar is instantiated in the cloud and starts monitoring connectivity condition together with the MPG's location information. The Avatar interacts with the leader node to configure monitoring requirements as well as to collect monitoring updates.

\subsubsection{Selective resource monitoring}

Clarinet provides a configuration dashboard that allows a user to configure resource monitoring. Such configuration is important for the performance of the MPG and privacy of the user. Continuous invocation of monitoring requires significant amount of energy as well as the acquisition of finegrained contextual information (e.g., GPS location), which might threaten a mobile user's privacy. Clarinet provides the interface for mobile users or network administrators to opt in/out on certain monitoring activities in the MPG.

\subsubsection{Remote resource sharing}

Clarinet also enables the interaction between Avatar instances of different mobile users and the interaction between the cloud and the network infrastructure. The interaction between Avatar instances can facilitate physical interaction among MPGs. For example, if two mobile users share one file, each user's Avatar can select which connectivity should be used for the sharing, as opposed to each user manually changing their connectivity.

Clarinet exposes APIs for application developers or resource owners to leverage the monitoring infrastructure. For instance, through the APIs, an application can obtain the user's connectivity history in certain areas for content prefetching, and embedded computing resource such as display or sensors can register their resource to the MPG for sharing.

\subsection{Current Status}

Figure 3 shows the current prototype of Clarinet. We are developing the core components using open-source based systems. The implementation consists of client/server software and message transport protocols. A Clarinet client, running on every device in the MPG, has been implemented on top of Android OS 2.3 as a service. The MPG Manager in the client coordinates the local devices of a user, the Monitor

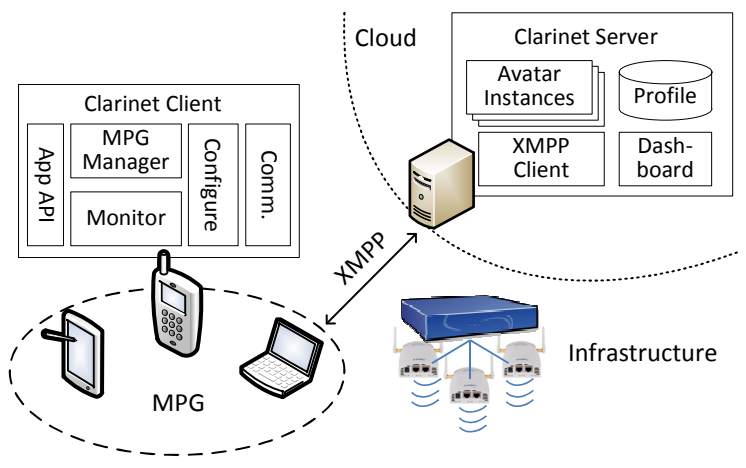

Figure 3: The Clarinet design.

periodically senses and collects resource status, the Configure module provides an interface for a user to configure what resource needs to be monitored and interacts with a server to update the configuration, the Comm module transports control/update information between the MPG and a server in the cloud, and App API allows a third-party application to access Clarinet resource information.

In the server side, we use an open-fire server [13] to process Carmen messages and employ open-source web framework, called Ruby [14] to instantiate and maintain the process of each MPG's Avatar. Further, we use XMPP [15] for the communication between the MPG and the cloud. The Clarinet server maintains the Avatar instance for each MPG, and each MPG's history and connectivity status is stored in the database (Profile). The Dashboard provides an aggregate and customized view on connectivity history over certain geographical areas per Avatar.

\section{RELATED WORK}

Due to resource constraints on the mobile devices, many research threads are considering how to augment the mobile device's capability with resources found locally or in the cloud. Partitioning computation-intensive applications (e.g., object recognition) across the mobile, local networks, and remote clouds at run time are proposed in [16, 17, 6, 18]. Virtual machine based approaches have also been proposed for secure and bandwidth-efficient use of other existing resources [19]. Although our Carmen architecture is similar to their approaches in leveraging the resource of the cloud for the mobile devices, our work mainly focuses on providing seamless network connectivity for a group of mobile devices by leveraging global presence of cloud and its deep integration with the mobile devices and the network infrastructure under multi-dimensional mobility.

Meraki has recently developed a cloud-managed wireless LAN controller to manage physically distributed enterprise Wireless LANs [20]. Their approach provides central network management capability of entire wireless network infrastructure using the power of cloud's global accessibility. By contrast, our work aims to help each user's connectivity management across heterogeneous networks that mobile users encounter.

Mobile-based sensing has recently drawn much attention. mCrowd [21] allows users to participate in search, survey task, and environmental monitoring. Open mobile health [22] has been focusing on monitoring personal health using mo- 
bile devices, and it also allows crowd to participate in data collection for environment and health-related data. Finally, funf [23] is an open mobile sensing framework based on Android OS. It provides useful APIs for developers to integrate smartphone-based sensing capability based on the concept of 'data probe'. All of the previous work have focused on monitoring specific aspect of mobile user's behavior on a single device, but Clarinet differs from them in its focus on mobile connectivity across multiple devices and deep integration with the cloud and the network infrastructure.

\section{CONCLUDING REMARKS}

We presented Carmen, a novel cloud-centric networking architecture for seamless and pervasive bonding between a mobile user and a cloud. The Carmen architecture allows a user to get efficient and timely assistance in resolving connectivity issues from a cloud-based connectivity assistant, Avatar. We have identified key challenges induced by multiple dimensions of mobility - access networks, devices, and personas - and proposed novel design principles to provide rich and seamless networking experience to mobile users. In addition, we have shown that it is instrumental to take a holistic approach across mobile devices, network infrastructure, and the cloud to address challenges under multi-dimensional mobility. As a proof-of-concept, we have shown our work-in-progress, Clarinet, that provides mobile resource-monitoring infrastructure with the help of the cloud. Our ongoing work includes addressing technical challenges that we have identified in the Carmen architecture. Further, we are continuing to develop Clarinet to enable close interaction among Avatar instances as well as between Avatars and the network infrastructure to better support rich mobile networking experience.

\section{Acknowledgement}

Authors thank Sujata Banerjee, Jack Brassil, Ran-Fun Chiu, Puneet Sharma, David Lee, Jeongkeun Lee, and Junxing Xie for their contribution and comments on the Carmen architecture, as part of mobility research initiative at HP Labs.

\section{REFERENCES}

[1] OnLive Video Game. Available from: http://www.onlive.com/mobile.

[2] Dropbox. Available from: http://www.dropbox.com/.

[3] Digital Living Network Alliance, 2011. Available from: http://www.dlna.org/home.

[4] White Paper: LTE TDD Operator Business Case \& Adoption Forecast, 2011. Available from: http://www. heavyreading. com.

[5] Cisco Visual Networking Index, February 2011. Available from: http:
//wWw.cisco.com/en/US/solutions/collateral/ns341/ ns525/ns537/ns705/ns827/white_paper_c11-520862.html.

[6] M. Satyanarayanan, P. Bahl, R. Caceres, N. Davies. The Case for VM-Based Cloudlets in Mobile Computing. IEEE Journal of Pervasive Computing, 8(4):14-23, October 2009.

[7] H. Hsieh, K.-H. Kim, Y. Zhu, and R. Sivakumar. A receiver-centric transport protocol for mobile hosts with heterogeneous wireless interfaces. In Proceedings of 9th ACM International Conference on Mobile Computing and Networking (MobiCom'03), San Diego, California, September 2003.

[8] C.Perkins. IP Mobility Support for IPv4. Internet Request For Comments3220, January 2002.

[9] K.-H. Kim and K. G. Shin. Improving TCP Performance over Wireless Networks with Collaborative Multi-homed Mobile Hosts. In Proceedings of 3th International Conference on Mobile Systems, Applications, and Services (MobiSys'05), June 2005.

[10] Puneet Sharma, Sung-Ju Lee, Jack Brassil, and Kang G. Shin. Aggregating bandwidth for multihomed mobile collaborative communities. IEEE Transactions on Mobile Computing, 6(3):280-296, 2007.

[11] The Future of Hotspots: Making Wi-Fi as Secure and Easy to Use as Cellular, 2011. Available from: http://www.cisco.com/en/US/solutions/collateral/ ns341/ns524/ns673/white_paper_c11-649337.html.

[12] Open Network Foundation, 2011. Available from: http://www.opennetworking.org.

[13] Openfire. Available from: http://www.igniterealtime.org/projects/openfigure.

[14] Ruby on Rails. Available from: http://rubyonrails.org.

[15] XMPP Standards Foundation. Available from: http://xmpp.org.

[16] A. Michael et al. Above the Clouds: A Berkeley View of Cloud Computing. Communications of the ACM, 53(4):50-58, April 2010.

[17] E. Cuervo, A. Balasubramanian, D. Cho, A. Wolman, S. Saroiu, R. Chandra, and P. Bahl. MAUI: Making Smartphones Last Longer with Code Offload. In Proc. of ACM MobiSys, June 2010.

[18] M. Ra, A. Sheth, L. Mummert, P. Pillai, D. Wetherall, and R. Govindan. Odessa: Enabling Interactive Perception Applications on Mobile Devices. In Proceedings of ACM MobiSys 2011.

[19] A. Wolbach, J. Harkes, S. Chellappa, and M. Satyanarayanan. Transient Customization of Mobile Computing Infrastructure. In Proc. of MobiVirt, June 2008.

[20] Meraki Cloud-Hosted Wireless LAN Controller, 2011. Available from: http://meraki.com/products/wireless/ enterprise-cloud-controller.

[21] mCrowd: Mobile Crowdsourcing, 2010. Available from: http://crowd.cs.umass.edu.

[22] Open mHealth, 2012. Available from: http://openmhealth.org.

[23] funf: Open Sensing Framework, MIT Media Lab, 2011. Available from: http://www.funf.org. 